Tárraga-Mínguez, R., Sanz-Cervera, P., Pastor-Cerezuela, G. \& Fernández-Andrés, M.I. (2017). Análisis de la autoeficacia percibida en el uso de las TIC de futuros maestros y maestras de Educación Infantil y Educación Primaria. Revista Electrónica Interuniversitaria de Formación del Profesorado, 20 (3), 107-116. DOI: http://dx.doi.org/10.6018/reifop.20.3.263901

\title{
Análisis de la autoeficacia percibida en el uso de las TIC de futuros maestros y maestras de Educación Infantil y Educación Primaria
}

Raúl Tárraga-Mínguez ${ }^{(1)}$, Pilar Sanz-Cervera ${ }^{(1)}$, Gemma Pastor-Cerezuela ${ }^{(2)}, M^{a}$ Inmaculada Fernández-Andrés ${ }^{(3)}$.

'Dpto. Didáctica y Organización Escolar. Universitat de València; ${ }^{2} D p t o$. Psicología Básica. Universitat de València; ${ }^{3}$ Dpto. Psicología Evolutiva y de la Educación. Universitat de València. ${ }^{(3)}$

\section{Resumen}

La formación del profesorado en el uso de las TIC es un aspecto importante para conseguir una verdadera integración de las TIC en la educación. Según el modelo TPACK, esta formación no debe limitarse a contemplar de manera separada los aspectos relacionados con las TIC, el contenido curricular y la metodología. En el presente estudio se explora la percepción de autoeficacia en cuanto al uso de las TIC en el aula de una muestra de 107 estudiantes de los grados de Maestro/a en Educación Infantil y en Educación Primaria. Para ello se emplea una traducción del cuestionario para la autoevaluación de las competencias TIC elaborado por Tondeur et al. (2016). Los resultados del estudio muestran que los estudiantes de Magisterio se perciben a sí mismos como más competentes en el uso de las TIC que en la toma de decisiones pedagógicas relacionadas con dicho uso. Además, se halló una correlación estadísticamente significativa entre las horas de formación recibidas en el uso educativo de las TIC y la percepción de autoeficacia en este mismo uso educativo de las TIC. Estos resultados conllevan algunas implicaciones en la toma de decisiones respecto a la formación del profesorado.

\section{Palabras clave}

Autoeficacia percibida; formación del profesorado; modelo TPACK; TIC 


\title{
Analysis of the perceived self-efficacy in the use of ICT of pre- service primary and preschool teachers
}

\begin{abstract}
Teacher training in the use of ICT is an important issue for the integration of ICT in education. According to the TPACK model, this training should not be limited to contemplate separately aspects of ICT, curriculum content and methodology. The present study explores the self-efficacy of ICT use in a sample of 107 pre-service primary and preschool teachers. A translation of the questionnaire for self-assessment of ICT competences developed by Tondeur et al (2016) was used. Results show that pre-service teachers perceive themselves as more competent in the use of ICT, compared to making educational decisions related to such use. In addition, a statistically significant correlation between the hours of training received in the educational use of ICT and the perception of self-efficacy in this educational use of ICT was found. These results have some implications in decision-making regarding teacher training.
\end{abstract}

\section{Key words}

ICT; perceived self-efficacy; teacher training; TPACK model

\section{Introducción}

Actualmente no es ninguna novedad y prácticamente nadie pone en duda que las Tecnologías de la Información y la Comunicación (TIC) son un elemento que juega un papel importante en la educación. En las aulas hay presentes medios tecnológicos que eran impensables hace unas pocas décadas pero que ahora se han convertido en objetos cotidianos (ordenadores, pizarras digitales, proyectores, etc.)

Sin embargo, no está claro que la formación del profesorado en el uso técnico y pedagógico de las TIC se haya desarrollado a la misma velocidad que han llegado estos recursos, ya que mientras que la presencia en el aula de una pizarra digital es un hecho concreto que puede estar totalmente ausente un día y presente al siguiente, la formación del profesorado es una cuestión más compleja que requiere el aprendizaje de conocimientos técnicos, cambios en la metodología y las estrategias de enseñanza seguidas y que el profesorado confíe en los nuevos medios para mejorar su práctica. La formación del profesorado por tanto demanda un tiempo razonable para adaptarse a las metodologías de enseñanza derivadas de los recursos TIC (Ertmer y Ottenbreit-Leftwich, 2010).

Esta formación no debe limitarse tan solo al conocimiento técnico relativo al funcionamiento de ordenadores, programas informáticos, pizarras digitales u otras herramientas TIC, sino que debe también hacer hincapié en los aspectos pedagógicos, relativos a cómo incorporar las herramientas TIC a la metodología del aula de manera que realmente enriquezcan el aprendizaje.

\subsection{El modelo TPACK de Koheler y Mishra (2006)}

Koheler y Mishra (2006) han desarrollado precisamente un modelo sobre tecnología educativa en el que estudian la intersección entre contenido curricular, conocimiento pedagógico y conocimiento tecnológico: el modelo TPACK (Technological Pedagogical Content Knowledge). 
En este modelo, los autores señalan la importancia de que los diferentes elementos que lo componen, es decir, el contenido curricular, el conocimiento pedagógico y el conocimiento tecnológico, se conciban de un modo global, prestando atención a las intersecciones de estos elementos para de este modo llegar a concebir el uso de las TIC en la educación como un proceso complejo, que precisa de diferentes disciplinas para llegar a materializarse con la suficiente calidad. Así, según este modelo, el profesorado necesita no solo ser experto en el contenido curricular que es objeto de enseñanza, sino que también debe ser un experto en metodología de enseñanza, ser capaz de utilizar con soltura los medios tecnológicos de que disponga en su aula, y lo que probablemente es más importante, necesita saber conjugar estos tres tipos de conocimiento para ponerlos al servicio de los procesos de enseñanza en el aula.

El modelo de Koehler y Mishra (2009) ha generado un importante impacto en la concepción del uso de las TIC en la educación, ya que ha ayudado a superar la concepción dicotómica artificial en la que la pedagogía y la tecnología se concebían como disciplinas totalmente aisladas ( $y$ en ocasiones incluso confrontadas).

Lo importante son las intersecciones entre TIC, contenido y metodología, de manera que se buscan los puntos en común en que estos tres elementos pueden incidir en la mejora educativa, en lugar de aislar dichos elementos, ignorando que en realidad el uso que se haga en el aula de las TIC es una decisión básicamente de metodología, es decir, que no debe tomarse con otro criterio que el pedagógico.

\subsection{Investigaciones previas sobre las competencias TIC de los estudiantes de Magisterio}

Las investigaciones que se han desarrollado hasta el momento explorando cuál es el grado de autoeficacia percibida en el uso de las TIC, se han centrado fundamentalmente en profesorado en activo (Sigalés, Mominó, Meneses y Badía, 2008; Valverde, Garrido y Fernández, 2010; Suárez, Almerich, Gargallo y Aliaga, 2013).

En el caso de investigaciones que han tratado de estudiar las competencias en el uso de las TIC por parte de los estudiantes de Magisterio son relativamente escasas, aunque ofrecen información muy interesante.

Romero y Minelli (2011) en el artículo "La generación TIC se tambalea. Percepción del dominio de las TIC en estudiantes de Magisterio", cuestionaban la extendida creencia de que los estudiantes de Magisterio más jóvenes tenían un mayor nivel de competencias TIC que los estudiantes de Magisterio de mayor edad. Los resultados del estudio hallaron que no existía una relación estadísticamente significativa entre la edad de los participantes y su percepción acerca del dominio de las TIC. Según los autores "los estudiantes más jóvenes no mostraron, en líneas generales, las características que la literatura atribuye a la Generación Net, de hecho, algunos de los alumnos mayores mostraron una mejor percepción en el dominio de las TIC" (Romero y Minelli, 2011, p. 239). Esto es contradictorio con el obtenido por Suárez et al. (2013), realizado con profesorado en activo lo que parece sugerir que, si bien existe una diferencia en el grado de familiaridad con las TIC entre el profesorado en activo, esta diferencia parece diluirse cuando se trata de estudiantes del grado de Magisterio.

En otro estudio que trata de ahondar en la percepción de los estudiantes de Magisterio sobre sus competencias TIC, Gallego, Gámiz y Gutiérrez (2010) aplicaron un cuestionario a una muestra de estudiantes de Magisterio en el que precisamente indagaban sobre esta cuestión. Uno de los resultados que obtuvieron fue contrario al obtenido en la mayoría de estudios realizados con maestros en activo, y es que indican que los estudiantes perciben que poseen mayores competencias didácticas en comparación a sus competencias 
tecnológicas. Si bien probablemente Gallego et al. (2010) no realizan la distinción entre competencias tecnológicas y pedagógicas en el mismo sentido que se exponen en el modelo TPACK, sí es un dato a tener en cuenta y que invita a continuar investigando sobre el perfil de competencias TIC del profesorado.

En el ámbito internacional, también se encuentran artículos que analizan cuál es la competencia TIC en formación del profesorado. En esta línea, Sang, Valcke, van Braak y Tondeur (2010) llevaron a cabo una investigación con una muestra de estudiantes de Magisterio en universidades de China. En esta investigación sometieron a validación un modelo con la intención de identificar qué variables predicen el grado de integración de las TIC en la docencia. Los resultados indicaron que el grado de integración de las TIC se correlacionaba con las creencias del profesorado, especialmente sobre la enseñanza basada en el constructivismo, la autoeficacia relativa a aspectos de docencia, la autoeficacia relacionada con aspectos de las TIC y las actitudes hacia la integración de las TIC en la educación.

\subsection{Objetivos del estudio}

El objetivo general del presente estudio consiste en evaluar la autoeficacia percibida en el uso de las TIC en el aula en un grupo de estudiantes de los grados de Maestro/a en Educación Infantil y Maestro/a en Educación Primaria.

Este objetivo general se concreta en los siguientes objetivos específicos:

1. Analizar si existen diferencias en la autoeficacia percibida de los estudiantes en dos factores diferentes que incluye el cuestionario empleado: "competencias para apoyar a los estudiantes en el uso de las TIC en el aula" y "competencias para usar las TIC en el diseño instruccional".

2. Analizar si existe relación entre el número de horas de formación recibida directamente relacionada con las TIC y la autoeficacia percibida de los estudiantes para hacer uso de las TIC.

3. Analizar si existen diferencias en la autoeficacia percibida en el uso de las TIC entre los estudiantes del grado de Maestro/a en Educación Infantil y los estudiantes del grado de Maestro/a en Educación Primaria.

Consideramos que estos objetivos pueden contribuir a arrojar luz sobre el tema de la formación TIC del profesorado en tanto que:

- Se utiliza un instrumento basado en la valoración de los propios estudiantes sobre su propia autoeficacia y formación.

- El instrumento se adapta adecuadamente a la base del modelo TPACK, uno de los modelos que contemplan con mayor acierto las claves de la formación TIC del profesorado.

- Se realiza una comparación entre los estudiantes del Grado de Maestro/a en Educación Infantil y en Educación Primaria, un aspecto poco explorado hasta ahora y que es interesante, ya que los procesos educativos que se desarrollan en estas dos etapas tienen notables diferencias.

- Por último, el análisis de la cantidad de formación (cuantificada en horas) que están recibiendo los estudiantes de Magisterio puede ayudar a valorar si es suficiente para alcanzar las competencias TIC que se esperan de estos estudios. 


\section{Método}

\subsection{Participantes}

En el estudio participaron 107 estudiantes (82 mujeres, 25 hombres) de los grados universitarios de Maestro/a en Educación Infantil y Maestro/a en Educación Primaria en la Universidad de Valencia, con edades comprendidas entre los 19 y 35 años. En la tabla 1 se incluyen las características demográficas de los participantes.

Tabla 1.

Información demográfica de los participantes.

\begin{tabular}{lll}
\hline \multirow{2}{*}{ Sexo } & Mujeres & $82(76.6 \%)$ \\
\cline { 2 - 3 } & Hombres & $25(23.4 \%)$ \\
\hline \multirow{2}{*}{ Grado } & Educación Infantil & $33(30.8 \%)$ \\
\cline { 2 - 3 } & Educación Primaria & $72(63.6 \%)$ \\
\hline \multirow{2}{*}{ Edad } & Media (DT) & $20.41(2.32)$ \\
\hline
\end{tabular}

\subsection{Instrumento}

Para la realización del presente estudio, el autor del presente trabajo tradujo del inglés al castellano el cuestionario para la autoevaluación de las competencias TIC elaborado por Tondeur et al. (2016) siguiendo el método de traducción inversa por Posteriormente, un estudiante de filología cuya lengua materna era el inglés, tradujo el cuestionario de castellano a inglés. Finalmente, se analizaron las diferencias entre la versión del cuestionario original y la versión traducida por el estudiante'.

Se trata de un cuestionario de 19 ítems tipo Likert en los que se solicita a los participantes que autoevalúen su capacidad para llevar a cabo diferentes acciones relacionadas con el uso de las TIC en el aula. Por otro lado, el instrumento consta de 2 factores:

El factor 1 comprende los primeros 11 ítems, en los que se evalúan las competencias para apoyar a los estudiantes en el uso de las TIC en el aula. El factor 2 comprende los 8 ítems restantes, en los cuales se evalúan las competencias para usar las TIC en el diseño instruccional.

Además, se solicitaron 3 datos demográficos básicos: edad, sexo y grado que cursaba cada estudiante.

Finalmente, se solicitó también a los participantes que respondieran aproximadamente a cuántas horas se había dedicado en clase, durante su formación inicial, a tratar cuestiones directamente relacionadas con el uso de las TIC en el aula.

\subsection{Procedimiento}

Los participantes cumplimentaron voluntariamente el cuestionario. Los estudiantes fueron informados de que las respuestas serían tratadas de forma anónima y que no tendría ninguna repercusión en la calificación de la asignatura. Una vez finalizado este proceso, se codificaron las respuestas en el programa estadístico SPSS 22.0 y se llevaron a cabo los análisis descritos en la sección de resultados.

\footnotetext{
${ }^{1}$ Los ítems en que hubo diferencias fueron discutidos hasta que se llegó a un acuerdo para que la traducción al castellano fuera lo más fiel posible a la versión original, pero al mismo tiempo el contenido se adaptara al contexto español.
} 


\section{Resultados}

\subsection{Resultados del objetivo 1}

Al analizar si existen diferencias en la autoeficacia percibida de los estudiantes en los dos factores del cuestionario empleado: "competencias para apoyar a los estudiantes en el uso de las TIC en el aula" y "competencias para usar las TIC en el diseño instruccional", la puntuación obtenida en el factor 1 fue de 3.46 ( $D T=.62)$, mientras que la puntuación obtenida en el factor 2 fue de $3.09(D T=.76)$.

Se procedió a realizar una prueba $t$ de Student con un resultado de $t_{(106)}=8,93 ; p=.000$, lo que indica que la diferencia entre los factores sí es estadísticamente significativa.

\subsection{Resultados del objetivo 2}

Ante la pregunta: Aproximadamente, ¿cuántas horas calculas que se ha dedicado en clase en estos dos cursos de tu formación inicial al uso de las TIC en el aula?, los estudiantes ofrecieron respuestas que oscilaron entre las 0 y las 20 horas. El valor medio de las respuestas fue $2.12 \mathrm{~h}(\mathrm{DT}=3.29)$.

Se realizó un análisis de correlación de Pearson para comprobar si existía una relación estadísticamente significativa entre el número de horas que habían recibido formación en TIC y los resultados del cuestionario. Los resultados indicaron que esta correlación sí era estadísticamente significativa, tanto para el factor $1(r=.39, p=.000)$ como para el factor 2 $(r=.36, p=.000)$.

\subsection{Resultados del objetivo 3}

Para analizar si existe diferencias estadísticamente significativas en la autoeficacia percibida en el uso de las TIC entre los estudiantes del grado de Maestro/a en Educación Infantil y los estudiantes del grado de Maestro/a en Educación Primaria, se realizaron pruebas ANOVAs. No se encontraron diferencias estadísticamente significativas ni para el factor $1\left(F_{(1,105)}=1.42\right.$, $\left.p=.236, \eta_{p}^{2}=.013\right)$, ni para el factor $2\left(F_{(1,105)}=1.01, p=.318, \eta_{p}^{2}=.010\right)$.

\section{Conclusión}

Los resultados obtenidos en el presente trabajo nos llevan a al menos 3 conclusiones interesantes:

En primer lugar, los resultados muestran que los estudiantes se consideran a sí mismos más capacitados para las tareas más directamente relacionadas con el uso en sí de las TIC que para las tareas relacionadas con los aspectos pedagógicos de estas TIC.

Este resultado parece sugerir que los futuros maestros y maestras confían razonablemente en su capacidad técnica para utilizar correctamente las TIC y para propiciar que sus estudiantes las utilicen. Sin embargo, se perciben a sí mismos como menos competentes para tomar decisiones pedagógicas relacionadas con este uso de las TIC en el aula. Por ejemplo, los ítems que obtuvieron peor puntuación hacían referencia a rediseñar aplicaciones TIC específicas para situaciones educativas concretas, educar a los estudiantes para utilizar las TIC teniendo en cuenta aspectos como los factores ergonómicos, la propiedad intelectual, etc., o incluso el hecho de seleccionar aplicaciones TIC específicas para situaciones educativas concretas.

En conjunto, los resultados dibujan a futuros maestros que confían en su capacidad para llevar a cabo actividades que implican un uso activo de las TIC, pero se sienten menos 
capaces de tomar las decisiones pedagógicas que les deben llevar a planificar y evaluar esas actividades.

Aunque este segundo aspecto es hasta cierto punto comprensible, dado que los estudiantes se encuentran todavía en su formación inicial y razonablemente pueden percibir que todavía queda mucho por recorrer, este resultado parece indicar que si se toma el modelo TPACK como base para interpretar la formación en el uso de las TIC del profesorado, no parece que haya una predisposición a una verdadera integración de las TIC en la educación.

El resultado se alinea con el obtenido previamente comentado del estudio de Suárez et al. (2013), en que el profesorado en activo se consideraba mejor formado en cuanto a competencias tecnológicas que pedagógicas.

Además, debemos tener en cuenta que los participantes son estudiantes universitarios con una media de edad de 20.41 años. Es decir, pertenecen a una generación nacida en el año 1995 que probablemente ha tenido durante muchos años acceso a ordenadores, Internet y tecnología móvil, y las han utilizado en su día a día por motivos de ocio y estudio. Ello explica probablemente que el uso técnico de las herramientas TIC no sea percibido como una dificultad para estos estudiantes, sino que se sienten seguros de que serán capaces de utilizar con soltura, ya que pertenecen a lo que Prensky (2001) denominó nativos digitales.

En cualquier caso, no debemos olvidar el resultado obtenido por Romero y Minelli (2011), en el que llegaban literalmente a la conclusión de que "la generación Net se tambalea", por lo que debemos tomar con cierta cautela la afirmación del párrafo anterior.

En segundo lugar, los resultados del presente estudio muestran que sí existe una estrecha relación entre las horas de formación recibidas directamente relacionadas con las TIC, con la autoeficacia percibida de los futuros maestros y maestras para hacer uso de las TIC en el aula.

Los resultados muestran que esta relación es intensa tanto para los aspectos relacionados con el uso de las TIC en el aula como para los aspectos relacionados con las decisiones pedagógicas relacionadas con el uso de estas TIC.

Estos resultados deben tomarse también con cautela, dado que en general las horas de formación directamente relacionadas con las TIC que han recibido hasta esta fase de su formación inicial los futuros maestros y maestras ha sido todavía escasa (los estudiantes afirman haber dedicado hasta el momento una media de 2.12 horas durante el más de un curso y medio que han estudiado hasta el momento). Sin embargo, pese a lo poco intensa que ha sido esta formación hasta el momento, dicha formación parece encontrarse estrechamente relacionada con el grado de autoeficacia percibida. Relacionado con este objetivo, un aspecto preocupante de los resultados obtenidos en este estudio es el escaso número de horas que los estudiantes afirman haber recibido hasta el momento relacionado con el uso de las TIC en el aula. Este resultado es coherente con el hecho de que los estudiantes no han cursado ninguna asignatura directamente relacionada con el uso de las TIC. Pese a ello, resulta preocupante que en el resto de asignaturas que han cursado hayan dedicado poco más de una sesión al uso de las TIC en el aula.

Si analizamos los planes de estudio de los dos grados de Maestro/a en la Universidad de Valencia, vemos que en el caso del grado de Educación Infantil no existe durante todo el plan de estudios ninguna asignatura que en su título esté directamente relacionada con las TIC. En el caso del grado de Educación Primaria, vemos que no existe ninguna asignatura de este tipo que sea obligatoria para todos los estudiantes, aunque sí existe una mención de TIC, consistente en un itinerario de 5 asignaturas optativas en tercer y cuarto curso del 
grado que tienen precisamente a las TIC como eje central sobre el que giran todos los contenidos. Las asignaturas son: Educación y TIC, programario y maquinaria en contextos educativos, diseño de materiales educativos, TIC como recurso didáctico en artes y humanidades y TIC como recurso didáctico en ciencias y matemáticas. Este itinerario es cursado por aproximadamente 45 estudiantes de los cerca de 400 que cursan el grado en cada cohorte.

Por tanto, según este plan de estudios, ninguno de los estudiantes del grado de Educación Infantil y la gran mayoría de los estudiantes del grado de Educación Primaria cursará durante sus 4 años de formación inicial ninguna asignatura directamente relacionada con las TIC, lo que es un hecho preocupante, no porque los futuros maestros/as no vayan a estar formados en el uso de ordenadores u otros aparatos, sino porque probablemente no recibirán formación sobre los aspectos pedagógicos relacionados con las TIC.

A este respecto, una competencia que debe trabajarse en ambos grados según la información proporcionada en los propios planes de estudio de los títulos es: "utilizar con solvencia las tecnologías de la información y de la comunicación como herramientas de trabajo habituales" de lo que deducimos que estas competencias se deben trabajar transversalmente en diversas asignaturas, y no en una única asignatura.

Sin embargo, dado que ante la pregunta formulada al inicio del cuestionario: Aproximadamente, ¿cuántas horas calculas que se ha dedicado en clase en estos dos cursos de tu formación inicial al uso de las TIC en el aula?, la respuesta media de los estudiantes fue 2.12 horas, no podemos estar totalmente seguros de que dichas competencias se estén trabajando de manera efectiva, al menos en las asignaturas de los dos primeros cursos que los estudiantes han cursado hasta el momento.

En tercer lugar, al explorar si existían diferencias en la autoeficacia percibida por los estudiantes, los resultados mostraron que la diferencia entre ambos grupos de estudiantes no fue estadísticamente significativa. Este resultado es coherente, en tanto que, durante los dos primeros cursos de ambos grados, el currículum cursado coincide en un elevado porcentaje de las materias cursadas (tan solo hay 2 asignaturas diferentes para ambos grados en primer y segundo curso). Por tanto, pese a que probablemente el uso que se hará de las TIC en Educación Primaria y en Educación Infantil será bastante diferente, el bagaje formativo hasta el momento de los estudiantes de ambos grados les ha llevado a percibir una autoeficacia de sus propias capacidades similar para ambos grupos de estudiantes.

En cualquier caso, este resultado nos sugiere que la autoeficacia percibida respecto al uso de las TIC de los estudiantes de Magisterio sí está estrechamente relacionada con las horas de formación en esta materia, pero no lo está tanto con otros aspectos que probablemente diferencian a los estudiantes del grado de Educación Primaria y de Educación Infantil, como las motivaciones respecto a la profesión, las expectativas de cómo trabajar en el aula en el futuro, etc.

\subsection{Limitaciones del estudio}

El presente trabajo presenta algunas limitaciones que conviene tener en cuenta para interpretar los resultados con la debida cautela.

En primer lugar, la muestra empleada en el presente estudio ha sido reducida (107 estudiantes) y no es representativa de toda la población de estudiantes de Magisterio de la Universidad de Valencia (que asciende a aproximadamente unos 750 estudiantes). Además, la muestra no ha sido seleccionada mediante procedimientos aleatorizados, sino que han participado estudiantes de 3 agrupaciones horarias (de las 17 que existen en la facultad). 
En segundo lugar, debemos tener en cuenta que en el momento de responder los cuestionarios, los estudiantes se encontraban iniciando el segundo semestre del segundo curso del grado, es decir, se encontraban cerca del ecuador de su formación inicial como maestros, por lo que los resultados no muestran cuál es el grado de autoeficacia que se forma tras terminar la formación inicial de los maestros, sino que únicamente nos muestran cómo es esta autoeficacia en un momento intermedio de dicha formación.

Finalmente, debemos tener en cuenta también que la información obtenida proviene únicamente de un cuestionario. Quizá la aplicación de otros instrumentos de recogida de información como entrevistas o grupos de discusión pudiera haber complementado esta información y haber enriquecido los resultados.

\subsection{Futuras líneas de investigación}

Tras realizar este estudio, se abren algunas nuevas preguntas de investigación que sería interesante explorar en el futuro. Por ejemplo, sería interesante evaluar los aspectos incluidos en el presente estudio al inicio y al final del grado de magisterio, para analizar cómo varía la autoeficacia percibida de los futuros maestros y maestras tras completar toda su formación inicial. De este modo podríamos tener un indicador que, de algún modo, evaluara el impacto de la formación inicial en cuestiones relacionadas con las TIC.

Igualmente, sería interesante también analizar las cuestiones incluidas en la presente investigación en muestras de maestros y maestras que actualmente se encuentran desarrollando su labor en la escuela. De este modo, podríamos comparar si existen diferencias entre los profesionales que actualmente hay en la escuela, que debido a una diferencia generacional han tenido menos contacto directo en su día a día con la tecnología, y los futuros maestros y maestras que sí han sido socializados en un uso más cotidiano de las TIC en su día a día.

En tercer lugar, también sería interesante consultar al profesorado que imparte docencia en los grados de Magisterio para averiguar de qué manera están trabajando en sus asignaturas el uso de las TIC. De esta manera tendríamos una respuesta que complementaría la percepción de los estudiantes y podríamos contrastar si en efecto, tal y como afirman los estudiantes, se dedica un tiempo muy escaso a los aspectos relacionados con las TIC y la docencia, o si por el contrario, los profesores sí abordan en sus respectivas asignaturas el uso docente de las TIC, pero de un modo transversal que los estudiantes no han sabido identificar al responder el cuestionario.

Finalmente, también sería interesante analizar cuál es la relación entre la edad de los estudiantes y su percepción de autoeficacia en competencia TIC, para de este modo tratar de aclarar si el resultado de Romero y Minelli (2011) se corrobora en otras muestras, o si bien el concepto de "nativos digitales" propuesto por Prensky (2001), continúa vigente

\subsection{Implicaciones prácticas}

Finalmente, el presente trabajo tiene algunas implicaciones prácticas:

En primer lugar, el presente trabajo nos ha ayudado a conocer cómo valoran los estudiantes su capacidad para utilizar las TIC en el aula y para tomar decisiones pedagógicas sobre el uso de estas TIC. Esta información es interesante para detectar carencias en la formación inicial del profesorado, y por tanto, para tomar decisiones en el tipo de formación inicial que se ofrece en los grados de Maestro/a. En concreto, la información obtenida en el trabajo sugiere que la formación que se ofrece a los estudiantes debe encaminarse no solo a mejorar las habilidades directamente relacionadas con las propias TIC, sino también a mejorar las cuestiones relacionadas con la toma de decisiones pedagógicas relacionadas con estas TIC. Este tipo de formación se situaría en la línea de la "realfabetización" del 
profesorado que proponía Gutiérrez (2008), y del modelo TPACK que proponen Koehler y Mishra (2009).

En segundo lugar, el trabajo puede ser útil también para tomar decisiones en las futuras modificaciones de los planes de estudio de los grados, en las que se abrirá una oportunidad para replantearse qué tipo de modelo de formación del profesorado en el uso de las TIC se desea adoptar.

\section{Referencias}

Ertmer, P. A., y Ottenbreit-Leftwich, A. T. (2010). Teacher Technology Change; How Knowledge, Confidence, Beliefs and Culture Intersect. Journal of Research on Technology in Education, 42 (3), 255-284.

Gallego, M. J., Gámiz, V., y Gutiérrez, E. (2010). El futuro docente ante las competencias en el uso de las tecnologías de la información y comunicación para enseñar. Revista Electrónica de Tecnología Educativa (EDUTEC), 34, 1-18.

Gutiérrez, A. (2008). Las TIC en la formación del maestro. "Realfabetización" digital del profesorado. Revista Interuniversitaria de Formación del Profesorado, 63, 191-206.

Koehler, M., y Mishra, P. (2009). What is technological pedagogical content knowledge (TPACK)? Contemporary Issues in Technology and Teacher Education, 9(1), 60-70.

Prensky, M. (2001). Nativos digitales, inmigrantes digitales. On The Horizon, 9(5). Recuperado en abril de 2016 de: http://files.educunab.webnode.cl/2000000625aba35bb22/Nativos-digitales-parte1.pdf

Romero, M., y Minelli, J. (2011). La generación net se tambalea: percepción del dominio de las TIC de estudiantes de magisterio. Revista Electrónica Teoría de la Educación: Educación y Cultura en la Sociedad de la Información, 12(2), 265-283.

Sang, G., Valcke, M., van Braak, J., y Tondeur, J. (2010). Student teachers' thinking processes and ICT integration: Predictors of prospective teaching behaviors with educational technology. Computers \& Education, 54(1), 103-112.

Sigalés, C., Mominó, J. M., Meneses, J., y Badía, A. (2008). La integración de Internet en la educación escolar española: situación actual y perspectivas de futuro. Barcelona: UOC.

Suárez, J. M., Almerich, G., Gargallo, B., y Aliaga, F. M. (2013). Las competencias del profesorado en TIC: estructura básica. Educación XX1, 16(1), 39-62.

Tondeur, J., Aesaert, K., Pynoo, B., Braak, J., Fraeyman, N., y Erstad, O. (2016). Developing a validated instrument to measure preservice teachers' ICT competencies: Meeting the demands of the 21st century. British Journal of Educational Technology. doi: 10.1111/bjet.12380

UNESCO (2011). UNESCO ICT competency framework for teachers. París: UNESCO. Recuperado de http://unesdoc.unesco.org/images/0021/002134/213475e.pdf en abril de 2016.

Valverde, J., Garrido, $M^{a}$ C., y Fernández, R. (2010). Enseñar y aprender con tecnologías: un modelo teórico para las buenas prácticas educativas con TIC. Revista Electrónica Teoría de la Educación: Educación y Cultura en la Sociedad de la Información, 11(1), 203229. 